RMD

Open

Rheumatic \&

Musculoskeletal

Diseases

\title{
Relationship between the gut and the spine: a pilot study of first- degree relatives of patients with ankylosing spondylitis
}

\author{
Amy S Kehl, ${ }^{1}$ Thomas J Learch, ${ }^{2}$ Dalin $\mathrm{Li}^{3}{ }^{3}$ Dermot P B McGovern, ${ }^{3}$ \\ Michael H Weisman ${ }^{4}$
}

To cite: Kehl AS, Learch TJ, Li D, et al. Relationship between the gut and the spine: a pilot study of first-degree relatives of patients with ankylosing spondylitis. RMD Open 2017;3:e000437. doi:10.1136/ rmdopen-2017-000437

- Prepublication history for this paper is available online. To view these files please visit the journal online (http://dx. doi. org/10.1136/rmdopen-2017000437).

Received 16 January 2017 Revised 26 May 2017 Accepted 28 May 2017

\section{(1) CrossMark}

${ }^{1}$ Department of Rheumatology, University of California Los Angeles, Los Angeles, California, USA

'Department of Radiology, Cedars-Sinai Medical Center, Los Angeles, California, USA ${ }^{3}$ F. Widjaja Inflammatory Bowel and Immunobiology Research Institute, Cedars-Sinai Medical Center, Los Angeles, California, USA

${ }^{4}$ Department of Rheumatology, Cedars-Sinai Medical Center, Los Angeles, California, USA

Correspondence to Professor Michael H Weisman; weisman@cshs.org

\section{INTRODUCTION}

Patients with ankylosing spondylitis (AS) have a high frequency of asymptomatic acute and chronic gut inflammation resembling inflammatory bowel disease (IBD), with as many as $60 \%$ displaying evidence of microscopic gut inflammation in the absence of gastrointestinal (GI) symptoms. ${ }^{1}$ Further, there is considerable overlap in susceptibility loci between AS and IBD but this pleiotropic phenomenon likely only explains part of the clinical co-occurrence. ${ }^{2}{ }^{3}$ Animal model data investigating molecular mechanisms point to a critical relationship between the gut and AS and the potential role of the gut in the pathogenesis of AS. ${ }^{45}$ Several markers are utilised in clinical practice to determine the likelihood that a patient has IBD, the most common of which is the faecal calprotectin which has an estimated sensitivity and specificity of upwards of $80 \%$ for identification of patients with IBD and thus serves as a very useful marker of gut inflammation. ${ }^{6}$ Similarly, various IBD-related antibodies including anti-Saccharomyces cerevisiae antibodies (ASCA), antineutrophil cytoplasmic antibodies (ANCA), anti-I2 (associated with anti-Pseudomonas activity), anti-Escherichia coli outer membrane porin $\mathrm{C}$ (anti-OmpC) and antiflagellin antibodies (anti-CBir1) have been shown to have very good utility in distinguishing individuals with IBD from healthy controls, particularly when these tests are used in combination and thus serve as useful serological biomarkers for IBD. ${ }^{7}$

Using the above biomarkers along with advanced imaging studies, this pilot study investigated if first-degree relatives (FDRs) of patients with AS, who themselves do not carry a diagnosis of AS or IBD, have evidence of mucosal dysregulation, subclinical gut or sacroiliac joint inflammation that could potentially precede the development of overt IBD or AS. We postulate that this group will provide insights into the relationship between the gut and AS.

\section{METHODS}

The investigators collaborate in a large AS and IBD consultation and research centre where patients with AS and IBD are seen regularly for clinical care and participate in longitudinal outcome studies. For purposes of this pilot study, we approached a convenience sample of patients with AS (all fulfilling New York criteria for the diagnosis of AS, including X-ray evidence of definite sacroiliitis) from our voluntary research registry and those seen for clinical care at our institution who had an FDR without a known clinical diagnosis of either AS or IBD. Thirty-two healthy FDRs between the ages of 18 and 57 years were subsequently recruited for this study.

FDRs were excluded if known or previously diagnosed AS, IBD, inflammatory arthritis or other concomitant rheumatic disease was present. The study protocol was approved by the Institutional Review Board (IRB) at Cedars-Sinai Medical Center.

Blood (20 cc) was collected from each FDR and tested by ELISA for the presence of IBD-related antibodies: ASCA, ANCA, anti-I2, anti-OmpC and anti-CBir1 as previously described..$^{8-11}$ A stool sample $(10 \mathrm{cc})$ was also collected to measure faecal calprotectin (PhiCal Calprotectin ELISA, Quest Diagnostics) and a stool aliquot was saved for subsequent investigations of the microbiome. ${ }^{12} 13$ 
Table 1 Demographic and clinical characteristics of the 32 FDR subjects

\begin{tabular}{ll}
\hline Demographic and clinical characteristics & $\begin{array}{l}\text { Number of } \\
\text { patients (\%) }\end{array}$ \\
\hline Age 18-29years & 8 \\
\hline Age 30-50years & 22 \\
\hline Age>50years & 2 \\
Female & $16(50 \%)$ \\
Relationship to proband=sibling & 25 \\
Relationship to proband=offspring & 4 \\
$\begin{array}{l}\text { Relationship to proband=both sibling and } \\
\text { offspring }\end{array}$ & 3 \\
\hline HLA-B27 positive & 13 \\
\hline
\end{tabular}

FDR, first-degree relative; HLA-B27, human leucocyte antigen B27.

The FDRs received questionnaires examining the presence of AS or other spondyloarthritis ( $\mathrm{SpA}$ ) phenotypes. Clinical data about AS symptoms and signs were obtained using the Toronto Axial Spondyloarthritis Questionnaire in $\mathrm{IBD}^{14}$ and all subjects were queried about the presence of any GI symptoms. Additionally, all participants were asked if they experienced back pain for greater than 6 weeks. If they answered yes, they were subsequently questioned about their non-steroidal anti-inflammatory drug use.

A musculoskeletal pelvic MRI was obtained on all subjects using Short Tau Inversion Recovery and T1 sequences to examine for the presence of axial SpA. These images were independently read and interpreted by a trained and certified musculoskeletal radiologist (TJL).

\section{Statistical analyses}

Two-sided $p$ values comparing faecal calprotectin levels between two groups were calculated using the Wilcoxon rank-sum test. The faecal calprotectin levels were evaluated in subjects with sacroiliitis compared with those subjects without sacroiliitis. The calprotectin levels were also evaluated in serology positive as compared with serology negative subjects. p Values less than 0.05 were considered statistically significant.

\section{RESULTS}

\section{Demographic characteristics}

Thirty-two FDRs without a known diagnosis of AS or IBD were recruited; demographic characteristics of FDRs are shown in table 1 . Subjects ranged from 18 to 57 years in age with equal numbers of males and females. Twenty-five of the subjects were siblings of patients diagnosed with AS, four were children of affected patients and three had both an affected sibling and an affected parent. Of the 32 recruited FDRs, 13 subjects $(41 \%)$ were human leucocyte antigen (HLA)-B27+. Three FDRs had both an affected sibling and parent, only one of which (subject
12) was HLA-B27+, and this subject also had an MRI indicating mild active sacroiliitis. All of the documented probands of these three FDR subjects were HLA-B27+. Seven probands were HLA-B27- as were their FDRs. Of the 25 HLA-B27+ probands, 13 FDRs were negative for HLA-B27.

\section{Clinical characteristics}

In our FDRs, one subject had a history of eye inflammation and one had a history of psoriasis. None of the FDRs had GI symptoms suggesting GI inflammation, although one reported a remote history of gastric ulceration. Nine subjects reported low back pain of any kind for at least 6 weeks in a row.

The GI and rheumatological manifestations of the FDRs are shown in table 2. Seven $(\sim 22 \%)$ of the 32 FDRs were positive for at least one IBD-associated serological marker. Six were anti-CBir1 positive and two had a positive ASCA antibody. None of the FDRs were positive for ANCA, anti-I2 or anti-OmpC. Of the seven found to have a positive IBD antibody, two were HLA-B27+ (29\%) compared with 11 of the 25 (44\%) who were antibody negative. None of the FDRs with a positive IBD antibody had a history of inflammatory back pain. One of the seven patients with a positive IBD antibody had an abnormal MRI with evidence of sacroiliitis (table 2, Subject 27). None of the seven patients with a positive IBD antibody had existing GI symptoms.

Five FDRs out of the total cohort had MRI evidence of sacroiliitis, as read and interpreted by a musculoskeletal radiologist (table 2). One FDR (Subject \#2) had minimal bone marrow oedema at the right sacroiliac joint, which was not present on consecutive slices and should not be considered positive. Of the five FDRs with MRI evidence of sacroiliitis, three were HLA-B27+. One of the patients with a positive IBD antibody also had evidence of bilateral sacroiliitis. Three of the nine FDRs who endorsed back pain had evidence of sacroiliitis by MRI; six did not have imaging evidence of sacroiliitis.

The faecal calprotectin value was numerically, but not statistically, higher in subjects with MRI findings of sacroiliitis $(74.65 \mathrm{mcg} / \mathrm{g})$ as compared with those subjects who did not have MRI evidence of sacroiliitis $(40.85 \mathrm{mcg} / \mathrm{g})$ $(p=0.18)$. The difference between the faecal calprotectin values in subjects with IBD antibodies $(54.64 \mathrm{mcg} / \mathrm{g}$ ) as compared with those without IBD antibodies (42.69 $\mathrm{mcg} / \mathrm{g}$ ) was numerically higher but not statistically significant $(p=0.63)$. The mean calprotectin value in patients without IBD antibodies and without MRI findings of sacroiliitis was $37.06 \mathrm{mcg} / \mathrm{g}$ (table 3 ).

\section{DISCUSSION}

Our pilot study revealed that a substantial proportion of otherwise healthy FDRs of patients with AS displayed a positive IBD antibody profile, specifically with respect to anti-CBirl and without clinical evidence of IBD, findings which are suggestive of mucosal dysregulation. This 
Table 2 Evidence of subclinical gut inflammation, mucosal dysregulation or MRI evidence indicative of sacroilitis in FDRs

\begin{tabular}{|c|c|c|c|c|c|c|}
\hline FDR & ASCA IgA & ASCA IgG & CBir1 & fCal (mcg/g) & MRI findings & HLA-B27 status \\
\hline Subject 2 & Negative & Negative & Negative & 109.4 (ULN) & $\begin{array}{l}\text { Minimal bone marrow oedema of } \\
\text { right sacroiliac joint present solely } \\
\text { on a single slice; not considered } \\
\text { positive }\end{array}$ & Positive \\
\hline Subject 4 & Negative & Positive & Negative & <15.6 (Negative) & Not present & Negative \\
\hline Subject 6 & Negative & Negative & Negative & $<15.6$ (Negative) & $\begin{array}{l}\text { Bilateral sacroiliitis with } \\
\text { inflammatory bone marrow } \\
\text { oedema }\end{array}$ & Negative \\
\hline Subject 8 & Positive & Positive & Positive & $157.3(\mathrm{ULN})$ & Not present & Negative \\
\hline Subject 11 & Negative & Negative & Positive & <15.6 (Negative) & Not present & Positive \\
\hline Subject 12 & Negative & Negative & Negative & 76.6 (Negative) & Mild active right sacroiliitis & Positive \\
\hline Subject 15 & Negative & Negative & Positive & $<15.6$ (Negative) & Not present & Negative \\
\hline Subject 16 & Negative & Negative & Negative & No sample available & $\begin{array}{l}\text { Small area of sclerosis of left } \\
\text { sacroiliac joint }\end{array}$ & Negative \\
\hline Subject 18 & Negative & Negative & Positive & 81 (Negative) & Not present & Negative \\
\hline Subject 20 & Negative & Negative & Positive & 28 (Negative) & Not present & Negative \\
\hline Subject 27 & Negative & Negative & Positive & 69.4 (Negative) & $\begin{array}{l}\text { Active sacroiliitis left inferior } \\
\text { sacroiliac joint, subtle changes } \\
\text { right sacroiliac joint suggest } \\
\text { chronic changes related } \\
\text { to sacroilitis but no active } \\
\text { inflammation }\end{array}$ & Positive \\
\hline Subject 28 & Negative & Negative & Negative & 137 (ULN) & Advanced bilateral sacroiliitis & Positive \\
\hline
\end{tabular}

ASCA, anti-Saccharomyces cerevisiae antibodies; CBir1, antiflagellin antibodies; fCal, faecal calprotectin; FDR, first-degree relative; HLA-B27, human leucocyte antigen B27; Ig, immunoglobulin; ULN, upper limit of normal.

is meaningful as the specificity of the majority of IBD antibodies is upwards of $80 \% .{ }^{15}$ Our data is in agreement with previously published data with regard to the presence of mucosal dysregulation in patients with established AS. ${ }^{9} 1617$ Approximately, $6.5 \%$ of patients with AS will develop overt clinical IBD during their lifetime and as many as $60 \%$ have evidence of microscopic gut inflammation without GI symptoms. ${ }^{1}$ A recent study found that a substantial proportion of patients with AS had evidence of clinical IBD, although analysis of the temporal pattern was not consistent with a causal relationship. ${ }^{18}$

Table 3 Mean faecal calprotectin values in FDRs according to IBD antibody and radiographic status

\begin{tabular}{lll}
\hline FDR status & $\begin{array}{l}\text { Mean fCal } \\
\text { (mcg/g) }\end{array}$ & p Value \\
\hline $\begin{array}{l}\text { Positive for sacroiliitis (SI) } \\
\text { findings in MRI }\end{array}$ & 74.65 & 0.18 \\
$\begin{array}{l}\text { Negative for sacroiliitis (SI) } \\
\text { findings in MRI }\end{array}$ & 40.85 & \\
$\begin{array}{l}\text { Positive for IBD antibodies } \\
\text { Negative for IBD antibodies }\end{array}$ & 54.64 & 0.63 \\
$\begin{array}{l}\text { Negative for SI and IBD } \\
\text { antibodies }\end{array}$ & 37.06 & \\
\hline
\end{tabular}

fCal, faecal calprotectin; FDR, first-degree relative; IBD,

inflammatory bowel disease.
A variety of antibodies directed against commensal flora are clinically relevant and characteristic of subgroups of IBD. ${ }^{19}$ These include ASCA, ANCA, anti-I2, anti-OmpC and anti-CBir1 antibodies. ${ }^{20}$ In prior studies from our group examining biomarkers of intestinal inflammation in AS subjects without clinical evidence of IBD, median anti-I2 response was greater in patients with AS, and abnormal faecal calprotectin levels were observed at a significantly higher frequency. ${ }^{9}{ }^{16}$ Further, levels of anti-Cbir1 and ANCA were elevated in patients with AS without clinical IBD. All of these studies point to the presence of mucosal dysregulation and subclinical gut inflammation in patients with AS. ${ }^{17}$

There is strong evidence that IBD serologies exist prior to the clinical onset and overt development of IBD, pointing to a prodromal state. ${ }^{19} 2122$ Examining this prodromal period in patients with $\mathrm{SpA}$ is important for disease prevention and will require additional studies.

A substantial proportion of our FDR cohort, 34.3\% $(n=11)$ had evidence of either subclinical IBD or MRI findings indicative of SpA. In addition, $15.6 \%(n=5)$ of FDRs had MRI evidence of SpA, suggesting that subclinical AS occurs in a healthy cohort of people at risk of AS that may never reach clinical significance. Our study which focused on the presence of IBD markers does extend and enhance the recent observations that many 
FDRs of HLA-B27+ probands exhibit clinical and/or imaging abnormalities suggestive of SpA. ${ }^{23}$

We observed that nearly one-quarter of healthy FDRs had evidence of a positive IBD antibody suggestive of mucosal dysregulation. In addition, the mean faecal calprotectin value, a marker of gut inflammation, was numerically but not statistically significantly higher in subjects with MRI findings of sacroiliitis than the mean faecal calprotectin value in patients without MRI findings of sacroiliitis $(74.65 \mathrm{mcg} / \mathrm{g}$ vs $40.85 \mathrm{mcg} / \mathrm{g})$. There was little overlap between our FDR study subjects with positive gut and those with sacroiliac joint abnormalities; neither group was limited solely to HLA-B27+ subjects.

Limitations exist in the interpretation of our data. Our cohort was small, limited to a convenience sample and did not examine all FDRs of each family. Conclusions of exact risk cannot be drawn. Much larger cohorts of patients examined in a cross-sectional manner would be needed to generate hypotheses to further test in longitudinal studies. In addition, the lack of a control group with healthy age-matched controls is a shortcoming of the study. However, this is a pilot study and national data regarding the prevalence of sacroiliac joint MRI abnormalities in the normal healthy population is limited. One small study did show minor abnormalities, usually limited to one slice, in approximately $20 \%$ of healthy normal subjects. ${ }^{24}$ In the current study, the frequency of positive at risk findings (either musculoskeletal or gut) in undiagnosed FDRs was most certainly high and justifies further studies to enable preventative interventional strategies.

Acknowledgements The authors would like to thank Tina Wu (Cedars-Sinai Medical Center) for patient recruitment and study coordination and Irina lanculescu (Cedars-Sinai Medical Center) for writing and editorial assistance.

Funding This study was supported in part by a Mallinckrodt Research Fellowship Grant awarded to ASK. Additional support was received from the Michelson Charitable Foundation, NIH/NCATS UL1TR0001881-01 and NIH P01AR052915.

Competing interests None declared.

Ethics approval Institutional Review Board.

Provenance and peer review Not commissioned; externally peer reviewed.

Open Access This is an Open Access article distributed in accordance with the Creative Commons Attribution Non Commercial (CC BY-NC 4.0) license, which permits others to distribute, remix, adapt, build upon this work non-commercially, and license their derivative works on different terms, provided the original work is properly cited and the use is non-commercial. See: http://creativecommons.org/ licenses/by-nc/4.0/

(c) Article author(s) (or their employer(s) unless otherwise stated in the text of the article) 2017. All rights reserved. No commercial use is permitted unless otherwise expressly granted.

\section{REFERENCES}

1. Jacques P, Van Praet L, Carron P, et al. Pathophysiology and role of the gastrointestinal system in spondyloarthritides. Rheum Dis Clin North Am 2012;38:569-82.
2. Jostins L, Ripke S, Weersma RK, et al.Host-microbe interactions have shaped the genetic architecture of inflammatory bowel disease. Nature 2012;491:119-24.

3. Danoy P, Pryce K, Hadler J, et al.Association of variants at $1 \mathrm{q} 32$ and STAT3 with ankylosing spondylitis suggests genetic overlap with Crohn's disease. PLoS Genet 2010;6:e1001195.

4. Rehaume LM, Mondot S, Aguirre de Cárcer D, et al. ZAP-70 genotype disrupts the relationship between microbiota and host, leading to spondyloarthritis and ileitis in SKG mice. Arthritis Rheumatol 2014;66:2780-92.

5. DeLay ML, Turner MJ, Klenk El, et al. HLA-B27 misfolding and the unfolded protein response augment interleukin-23 production and are associated with Th17 activation in transgenic rats. Arthritis Rheum 2009;60:2633-43.

6. von Roon AC, Karamountzos L, Purkayastha S, et al. Diagnostic precision of fecal calprotectin for inflammatory bowel disease and colorectal malignancy. Am J Gastroenterol 2007;102:803-13.

7. Lewis JD. The utility of biomarkers in the diagnosis and therapy of inflammatory bowel disease. Gastroenterology 2011;140:1817-26.

8. Targan SR, Landers CJ, Yang H, et al. Antibodies to CBir1 flagellin define a unique response that is associated independently with complicated Crohn's disease. Gastroenterology 2005;128:2020-8.

9. Mundwiler ML, Mei L, Landers CJ, et al. Inflammatory bowel disease serologies in ankylosing spondylitis patients: a pilot study. Arthritis Res Ther 2009;11:R177.

10. Aydin SZ, Atagunduz P, Temel M, et al. Anti-Saccharomyces cerevisiae antibodies (ASCA) in spondyloarthropathies: a reassessment. Rheumatology 2008;47:142-4.

11. Török HP, Glas J, Gruber R, et al. Inflammatory bowel disease-specific autoantibodies in HLA-B27-associated spondyloarthropathies: increased prevalence of ASCA and pANCA. Digestion 2004;70:49-54.

12. Konikoff MR, Denson LA. Role of fecal calprotectin as a biomarker of intestinal inflammation in inflammatory bowel disease. Inflamm Bowel Dis 2006;12:524-34.

13. Klingberg E, Carlsten H, Hilme E, et al. Calprotectin in ankylosing spondylitis - frequently elevated in feces, but normal in serum. Scand J Gastroenterol 2012;47:435-44.

14. Alnaqbi KA, Touma Z, Passalent L, et al. Development, sensibility, and reliability of the Toronto Axial Spondyloarthritis Questionnaire in inflammatory bowel disease. J Rheumatol 2013;40:1726-35.

15. Peyrin-Biroulet L, Standaert-Vitse A, Branche J, et al. IBD serological panels: facts and perspectives. Inflamm Bowel Dis 2007;13:1561-6.

16. Matzkies FG, Targan SR, Berel D, et al. Markers of intestinal inflammation in patients with ankylosing spondylitis: a pilot study. Arthritis Res Ther 2012;14:R261.

17. Wallis $\mathrm{D}$, Asaduzzaman $\mathrm{A}$, Weisman $\mathrm{M}$, et al. Elevated serum anti-flagellin antibodies implicate subclinical bowel inflammation in ankylosing spondylitis: an observational study. Arthritis Res Ther 2013;15:R166.

18. Inman RD, Lin A, Sherman P, et al. HLA-B27 influence on the gut microbiome (abstract). 2013.

19. van Schaik FD, Oldenburg B, Hart AR, et al. Serological markers predict inflammatory bowel disease years before the diagnosis. Gut 2013;62:683-8.

20. de Vries M, van der Horst-Bruinsma I, van Hoogstraten I, et al. pANCA, ASCA, and OmpC antibodies in patients with ankylosing spondylitis without inflammatory bowel disease. J Rheumatol 2010;37:2340-4.

21. Pimentel $\mathrm{M}$, Chang $\mathrm{M}$, Chow $\mathrm{EJ}$, et al. Identification of a prodromal period in Crohn's disease but not ulcerative colitis. Am J Gastroenterol 2000;95:3458-62.

22. Israeli E, Grotto I, Gilburd B, et al. Anti-Saccharomyces cerevisiae and antineutrophil cytoplasmic antibodies as predictors of inflammatory bowel disease. Gut 2005;54:1232-6.

23. Turina MC, de Winter JJ, Paramarta JE, et al. Clinical and imaging signs of spondyloarthritis in first-degree relatives of HLA-B27positive ankylosing spondylitis patients: the Pre-Spondyloarthritis (Pre-SpA) cohort study. Arthritis Rheumatol 2016;68:2444-55.

24. Weber $U$, Zubler V, Zhao Z, et al. Does spinal MRI add incremental diagnostic value to MRI of the sacroiliac joints alone in patients with non-radiographic axial spondyloarthritis? Ann Rheum Dis 2015;74:985-92. 